\title{
THE HARDY CLASS OF A BAZILEVIČ FUNCTION AND ITS DERIVATIVE
}

\author{
SANFORD S. MILLER
}

Abstract. The Bazilevič function $f(z)$ defined in $\Delta:|z|<1$ by $f(z) \equiv\left[\alpha \int_{0}^{z} P(\zeta) g(\zeta)^{\alpha} \zeta^{-1} d \zeta\right]^{1 / \alpha}$ where $g(\zeta)$ is starlike in $\Delta$, $P(\zeta)$ is regular with $\operatorname{Re} P(\zeta)>0$ in $\Delta$ and $\alpha>0$ is univalent. The class of such functions contains many of the special classes of univalent functions. The author determines the Hardy classes to which $f(z)$ and $f^{\prime}(z)$ belong. In addition if $f(z)=\sum_{0}^{\infty} a_{n} z^{n}$ the limiting value of $\left|a_{n}\right| / n$ is obtained.

If $g(z)$ is starlike (with respect to the origin) in $\Delta:|z|<1, P(z)$ is regular with $\operatorname{Re} P(z)>0$ in $\Delta, \beta$ is any real number and $\alpha>0$, then

$$
f(z)=\left[(\alpha+i \beta) \int_{0}^{z} P(\zeta) g(\zeta)^{\alpha} \zeta^{i \beta-1} d \zeta\right]^{1 /(\alpha+i \beta)}
$$

has been shown by Bazilevič [1] (see also Pommerenke [7]) to be a regular and univalent function in $\Delta$. The powers appearing in the formula are meant as principal values. We shall denote by $B(\alpha, \beta, P, g)$ the class of functions defined by (1).

Very little is known about $B(\alpha, \beta, P, g)$ in general, even though it is the largest known class of univalent functions defined by an explicit formula, and contains many of the heavily researched classes of univalent functions. In fact, we can easily see that for the classes of convex functions $C$, starlike functions $S^{*}$, spiral-like functions $S_{\gamma}$ and close-to-convex functions $K$, we have

$$
\begin{aligned}
& C=B(1,0,1, g), \\
& S^{*}=B\left(1,0, z g^{\prime} / g, g\right), \\
& S_{\gamma}=B\left(\cos \gamma, \sin \gamma, \cos \gamma z g^{\prime} / g+i \sin \gamma, g\right), \\
& K=B(1,0, P, g) .
\end{aligned}
$$

The Bieberbach conjecture remains unsettled for Bazilevič functions. J. Zamorski [9] has shown the conjecture valid for the class $B(1 / N, 0, P, g)$ where $N$ is a positive integer. $\mathrm{D}$. $\mathrm{K}$. Thomas [8] has shown that if $f(z)=\sum_{0}^{\infty} a_{n} z^{n}$ is in $B(\alpha, 0, P, g)$ and is bounded then $a_{n}=O(1 / n)$.

Presented to the Society, January 23, 1971; received by the editors August 12, 1970.

AMS 1970 subject classifications. Primary 30A32, 30A34, 30A78.

Key words and phrases. Univalent, Bazilevic functions, close-to-convex, Hardy class, coefficient growth condition, Cesaro summability. 
For $\lambda>0$, we say that a function $f(z)=\sum_{0}^{\infty} a_{n} z^{n}$ regular in $\Delta$ belongs to the Hardy class $H^{\lambda}$ if $\lim _{r \rightarrow 1^{-}} \int_{-\pi}^{\pi}\left|f\left(r e^{i \theta}\right)\right|^{\lambda} d \theta$ exists (and is finite). Eenigenburg and Keogh [2] have investigated the Hardy classes to which $f(z)$ and $f^{\prime}(z)$ belong for $f \in K=B(1,0, P, g)$. In this paper we carry out a similar investigation for the wider class $B(\alpha, 0, P, g)$, and deduce a growth condition for $a_{n}$.

In what follows, we denote by $k_{a, r}(z)$ any function of the form $a z\left(1-e^{-i \tau} z\right)^{-2}$, where $a$ is a complex constant and $\tau$ a real constant.

We require the following lemmas.

Lemma 1. If $f(z)$ is univalent, then $f(z) \in H^{\lambda}$, for all $\lambda<1 / 2$.

LEMMA 2. If $P(z)$ is regular and $\operatorname{Re} P(z)>0$ in $\Delta$, then $P(z) \in H^{\lambda}$ for all $\lambda<1$.

LEммA 3. If $g \in S^{*}$ and $g(z) \neq k_{a, \tau}(z)$ then there exists $\epsilon=\epsilon(g)>0$ such that $g(z) \in H^{1 / 2+e}$.

Lemma 4. If $f^{\prime}(z) \in H^{\lambda}(0<\lambda<1)$, then $f(z) \in H^{\lambda /(1-\lambda)}$.

LEMMA 5. If $f(z) \in H^{\lambda}(0<\lambda<1)$ and $f(z)=\sum_{0}^{\infty} a_{n} z^{n}$ then $a_{n}=o\left(n^{1 / \lambda-1}\right)$.

LEMMA 6. If $f(z)$ is univalent, $f(0)=0, \lambda>1 / 4$, and $[f(z)]^{\lambda}$ $=z^{\lambda} \sum_{0}^{\infty} b_{n} z^{n}$, then

$$
\lim _{n \rightarrow \infty} \frac{\left|b_{n}\right|}{n^{2 \lambda-1}}=\frac{\eta^{\lambda}}{\Gamma(2 \lambda)},
$$

where $\eta=\lim _{r \rightarrow 1^{-}}(1-r)^{2}\left[\max _{|z|-r}|f(z)|\right]$.

Lemma 1 is in [5, p. 214], Lemma 2 is well known, Lemma 3 is in [2, Theorem 6], Lemma 4 is in [3, Theorem 33], Lemma 5 is in [3, Theorem 28] and Lemma 6 is in $[4$, p. 104].

TheOREM 1. If $f \in B(\alpha, 0, P, g)$ with $g(z) \neq k_{a, r}(z)$ then there exists $\epsilon=\epsilon(f)>0$ such that $f(z) \in H^{1 / 2+\epsilon}$.

Proof. From (1) we have

$$
f(z)=\left[\alpha \int_{0}^{z} P(\zeta) g(\zeta)^{\alpha} \zeta^{-1} d \zeta\right]^{1 / \alpha}
$$

or

$$
f^{\prime}(z)=f(z)^{(1-\alpha)} g(z)^{\alpha} P(z) / z .
$$

If we let $F(z)=[f(z) / z]^{\alpha}$, then $F(z)$ is regular in $\Delta$ and satisfies 


$$
F^{\prime}(z)=\frac{\alpha f(z)^{\alpha-1} f^{\prime}(z)}{z^{\alpha}}-\frac{\alpha F(z)}{z} .
$$

On using (2), this differential equation becomes

$$
F^{\prime}(z)=\frac{\alpha g(z)^{\alpha} P(z)}{z^{\alpha+1}}-\frac{\alpha F(z)}{z},
$$

and if $0 \leqq \lambda \leqq 1$, then for $z=r e^{i \theta}(0<r<1)$ we obtain

$$
\begin{aligned}
I(r) \equiv & \int_{0}^{2 \pi}\left|F^{\prime}(z)\right|^{\lambda} d \theta \leqq \int_{0}^{2 \pi}\left|\alpha \frac{g(z)^{\alpha}}{z^{\alpha+1}} P(z)\right|^{\lambda} d \theta \\
& +\int_{0}^{2 \pi}\left|\frac{\alpha F(z)}{z}\right|^{\lambda} d \theta \equiv I_{1}(r)+I_{2}(r) .
\end{aligned}
$$

Now

$$
\begin{aligned}
I_{2}(r) & =\int_{0}^{2 \pi}\left|\frac{\alpha F(z)}{z}\right|^{\lambda} d \theta=\int_{0}^{2 \pi}\left|\frac{\alpha}{z}\left[\frac{f(z)}{z}\right]^{\alpha}\right|^{\lambda} d \theta \\
& =\frac{\alpha^{\lambda}}{r^{(\alpha+1) \lambda}} \int_{0}^{2 \pi}|f(z)|^{\alpha \lambda} d \theta,
\end{aligned}
$$

and since $f(z)$ is univalent, by Lemma $1, \lim _{r \rightarrow 1^{-}} I_{2}(r)$ exists provided that

$$
\alpha \lambda<1 / 2 .
$$

To investigate $I_{1}(r)$, we apply Hölder's inequality with conjugate indices $p$ and $q$. We have

$$
\begin{aligned}
\frac{r^{(\alpha+1) \lambda}}{\alpha^{\lambda}} I_{1}(r) & =\int_{0}^{2 \pi}|g(z)| \alpha \lambda|P(z)|^{\lambda} d \theta \\
& \leqq\left[\int_{0}^{2 \pi}|g(z)|^{\alpha \lambda p} d \theta\right]^{1 / p}\left[\int_{0}^{2 \pi}|P(z)|^{\lambda q} d \theta\right]^{1 / q} \\
& \equiv J_{1}(r) \cdot J_{2}(r) .
\end{aligned}
$$

By Lemma 3, $\lim _{r \rightarrow 1^{-}} J_{1}(r)$ exists if $\alpha \lambda p \leqq 1 / 2+\epsilon(g)=1 / 2+\epsilon$, and, by Lemma $2, \lim _{r \rightarrow 1^{-}} J_{2}(r)$ exists if $\lambda q<1$. Now using (3), (4) and (5), we see that $\lim _{r \rightarrow 1^{-}} I(r)$ exists provided that $\lambda<1 / 2 \alpha, 2 \alpha \lambda /(1+2 \epsilon)$ $\leqq 1 / p$ and $\lambda<1 / q$, where $1 / p+1 / q=1$ and $p>1$. This system of inequalities is satisfied with $p=1+(1+2 \epsilon) / 2 \alpha, q=1+2 \alpha /(1+2 \epsilon)$, and $\lambda \leqq(1+\epsilon) /(2 \alpha+1+\epsilon)$, where $\epsilon$ is chosen sufficiently small so that $(1+2 \epsilon) /(2 \alpha+1+2 \epsilon)<1 / 2 \alpha$. 
Hence we have shown that there exists $\epsilon>0$ such that $\lim _{r \rightarrow 1^{-}} I(r)$ exists if $\lambda \leqq(1+\epsilon) /(2 \alpha+1+\epsilon)$, i.e.

$$
F^{\prime}(z) \in H^{(1+\epsilon) /(2 \alpha+1+\epsilon)} \text {. }
$$

For $\lambda=(1+\epsilon) /(2 \alpha+1+\epsilon)$, we have $0<\lambda<1$ and

$$
\lambda /(1-\lambda)=(1+\epsilon) / 2 \alpha \text {. }
$$

By (6), (7) and Lemma 4 we therefore conclude that

$$
F(z) \in H^{(1+\epsilon) / 2 \alpha} .
$$

Since $f(z)=z[F(z)]^{1 / \alpha}$, we have

$$
\int_{0}^{2 \pi}|f(z)|^{\lambda} d \theta=r^{\lambda} \int_{0}^{2 \pi}|F(z)|^{\lambda / \alpha} d \theta
$$

and consequently from (8) we see that this last integral will be bounded provided that $\lambda / \alpha \leqq 1 / 2 \alpha+\epsilon / 2 \alpha$, i.e. $\lambda \leqq 1 / 2+\epsilon / 2$. Since $\epsilon$ is a function of $g(z)$ which is dependent on $f(z)$, we have shown that there exists $\epsilon=\epsilon(f)>0$ such that $f \in H^{1 / 2+\epsilon}$, and this completes the proof of the theorem.

The condition $g(z) \neq k_{a, r}(z)$ in the theorem is essential. This may be seen by taking $g(z)=k_{a, \tau}(z)$ and $P(z)=z k_{a, \tau}^{\prime}(z) / k_{a, \tau}(z)$. A simple calculation shows that for $f(z) \in B\left(\alpha, 0, z k_{a, \tau}^{\prime} / k_{a, \tau}, k_{a, \tau}\right)$ we have $f(z)$ $=k_{a, r}(z)$. Since

$$
\int_{0}^{2 \pi}\left|k_{a, \tau}(z)\right|^{1 / 2} d \theta=|a| r^{1 / 2} \int_{0}^{2 \pi} \frac{d \theta}{\left|1-r e^{i \theta}\right|} \geqq|a| \sqrt{ } 2 r^{1 / 2} \log \frac{1}{1-r},
$$

we see that $k_{a, \tau}(z) \notin H^{1 / 2}$.

By applying Lemma 5 we obtain the following corollary.

Corollary. If $f(z)=\sum_{0}^{\infty} a_{n} z^{n} \in B(\alpha, 0, P, g)$ and $g(z) \neq k_{a, \tau}(z)$, then there exists $0<\nu<1$ such that $a_{n}=o\left(n^{\nu}\right)$. In particular $\lim _{n \rightarrow \infty}\left|a_{n}\right| / n$ $=0$.

In the exceptional case $f(z) \in B\left(\alpha, 0, P, k_{a, \tau}\right)$ the limiting value of $\left|a_{n}\right| / n$ need not be zero and, as we shall see, depends on $P(z)$. Since $P(z)$ is regular in $\Delta$ and $\operatorname{Re} P(z)>0$ in $\Delta, P(z)$ has a Herglotz representation given by

$$
P(z)=|P(0)|\left[\cos \gamma \int_{0}^{2 \pi} \frac{1+z e^{-i t}}{1-z e^{-i t}} d \mu(t)+i \sin \gamma\right],
$$

where $P(0)=|P(0)| e^{i \gamma}, \mu(t)$ is nondecreasing and $\mu(2 \pi)-\mu(0)=1$. 
For the class $B\left(1,0, P, k_{a, \tau}\right)$ of close-to-convex functions, Eenigenburg and Keogh [2, Theorem 9] have shown that

$$
\left|a_{n}\right| / n \rightarrow|a P(0)|[\mu(\tau+0)-\mu(\tau-0)] \cos \gamma \text {. }
$$

We will prove a similar result for the wider class $B\left(\alpha, 0, P, k_{a, \tau}\right)$ when $\alpha \geqq 1 / 2$.

Theorem 2. If $f(z)=\sum_{1}^{\infty} a_{n} z^{n} \in B\left(\alpha, 0, P, k_{a, \tau}\right)$, where $\alpha \geqq 1 / 2$ and $P(z)$ is given by (9), then

$$
\left|a_{n}\right| / n \rightarrow|a|[|P(0)|(\mu(\tau+0)-\mu(\tau-0)) \cos \gamma]^{1 / \alpha} .
$$

In particular $\left|a_{n}\right| / n \rightarrow 0$ if and only if $\mu(t)$ is continuous at $\tau$.

Proof. If we let

$$
Q(z)=\int_{0}^{2 \pi} \frac{1+z e^{-i t}}{1-z e^{-i t}} d \mu(t)=\sum_{0}^{\infty} c_{n} z^{n} \quad\left(c_{0}=1\right),
$$

then

$$
\lim _{n \rightarrow \infty} \frac{1}{n} \sum_{0}^{n} c_{k} e^{i k \tau}=2[\mu(\tau+0)-\mu(\tau-0)] \equiv 2 S
$$

$[10$, p. $107,(9.3)]$. Hence the sequence $\left\{c_{k} e^{i k r}\right\}$ is summable $(C, 1)$, and consequently summable $(C, r)$ for $r \geqq 1$, i.e. if $A_{n}^{r}$ and $S_{n}^{r}$ are defined by the formulae

$$
\begin{gathered}
(1-\zeta)^{-r-1}=\sum_{0}^{\infty} A_{n}^{r} \zeta^{n}, \\
(1-\zeta)^{-r} \sum_{0}^{\infty} c_{n} e^{i n r} \zeta^{n}=\sum_{0}^{\infty} S_{n}^{r} \zeta^{n},
\end{gathered}
$$

then $S_{n}^{r} / A_{n}^{r} \rightarrow 2 S$ for $r \geqq 1[10$, p. 76$]$. Since

$$
A_{n}^{r}=\frac{\Gamma(r+n+1)}{\Gamma(n+1) \Gamma(r+1)}=\frac{n^{r}}{\Gamma(r+1)}\left\{1+O\left(\frac{1}{n}\right)\right\},
$$

we have

$$
A_{n}^{r-1} / n^{r} \rightarrow 0 \text { and } S_{n}^{r} / n^{r} \rightarrow 2 S / \Gamma(r+1)
$$

for $r \geqq 1$.

Since $f \in B\left(\alpha, 0, P, k_{a, \tau}\right)$ we have, by (9) and (10), 


$$
\begin{aligned}
f^{\alpha}(z) & =\alpha \int_{0}^{z} \frac{\left(k_{a, r}(\xi)\right)^{\alpha} P(\xi)}{\xi} d \xi \\
& =\alpha a^{\alpha}|P(0)| \int_{0}^{z} \xi^{\alpha-1}\left(1-e^{-i r \xi)^{-2 \alpha}}[Q(\xi) \cos \gamma+i \sin \gamma] d \xi,\right.
\end{aligned}
$$

or, writing $\zeta=e^{-i r \xi}$ and using (10),

$$
\begin{aligned}
f^{\alpha}(z)= & \alpha a^{\alpha}|P(0)| e^{i \alpha \tau} \\
& \cdot \int_{0}^{z e^{-i \tau}} \zeta^{\alpha-1}(1-\zeta)^{-2 \alpha}\left[\cos \gamma \sum_{0}^{\infty} c_{n} e^{i n \tau} \zeta^{n}+i \sin \gamma\right] d \zeta .
\end{aligned}
$$

Next using (11) and (12) and integrating, we obtain

$$
\begin{aligned}
f^{\alpha}(z) & =\alpha a^{\alpha}|P(0)|\left[\cos \gamma \sum_{0}^{\infty} \frac{S_{n}^{2 \alpha} e^{-i n \tau}}{n+\alpha} z^{n+\alpha}+i \sin \gamma \sum_{0}^{\infty} \frac{A_{n}^{2 \alpha-1} e^{-i n \tau}}{n+\alpha} z^{n+\alpha}\right] \\
& \equiv z^{\alpha} \sum_{0}^{\infty} b_{n} z^{n} .
\end{aligned}
$$

Now since

$$
\frac{(n+\alpha) b_{n} e^{i n \tau}}{n^{2 \alpha}}=\alpha a^{\alpha}|P(0)|\left[\frac{S_{n}^{2 \alpha}}{n^{2 \alpha}} \cos \gamma+i \frac{A_{n}^{2 \alpha-1}}{n^{2 \alpha}} \sin \gamma\right],
$$

from (13) with $2 \alpha \geqq 1$ we obtain

$$
\frac{(n+\alpha) b_{n} e^{i n \tau}}{n^{2 \alpha}} \rightarrow \frac{\alpha a^{\alpha}|P(0)| 2 S}{\Gamma(2 \alpha+1)} \cos \gamma
$$

which implies that

$$
\frac{\left|b_{n}\right|}{n^{2 \alpha-1}} \rightarrow \frac{2 \alpha|a|^{\alpha}|P(0)| S \cos \gamma}{\Gamma(2 \alpha+1)}=\frac{|a|^{\alpha}|P(0)| S \cos \gamma}{\Gamma(2 \alpha)} .
$$

On comparing this result with Lemma 6 for $\lambda=\alpha$, we see that

$$
\eta=\lim _{r \rightarrow 1^{-}}(1-r)^{2}\left[\max _{|z|=r}|f(z)|\right]=|a|[|P(0)| S \cos \gamma]^{1 / \alpha},
$$

and finally, applying Lemma 6 to the function $f(z)=\sum_{0}^{\infty} a_{n} z^{n}$ with $\lambda=1$, we have

$$
\lim _{n \rightarrow \infty}\left(\left|a_{n}\right| / n\right)=\eta / \Gamma(2)=|a|[|P(0)|[\mu(\tau+0)-\mu(\tau-0)] \cos \gamma]^{1 / \alpha},
$$

which completes the proof of the theorem. 
For a univalent function $f$ we know that $f \in H^{\lambda}$ for $\lambda<1 / 2$. However, $f^{\prime}$ need not belong to any $H^{\lambda}$ class [6]. We next show that this is not true for Bazilevič functions belonging to $B(\alpha, 0, P, g)$.

Theorem 3. If $f \in B(\alpha, 0, P, g)$ with $g(z) \neq k_{a, \tau}(z)$ then

(i) if $0<\alpha<1$, there exists $\delta=\delta(f)>0$ such that $f^{\prime}(z) \in H^{1 / 3+\delta}$;

(ii) if $1 \leqq \alpha$, there exists $\delta=\delta(f)>0$ such that $f^{\prime}(z) \in H^{1 /(2 \alpha+1)+\delta}$.

Proof. (i) On differentiating $f(z)=z F(z)^{1 / \alpha}$ we obtain

$$
f^{\prime}(z)=\frac{z}{\alpha} F(z)^{(1-\alpha) / \alpha} F^{\prime}(z)+F(z)^{1 / \alpha} .
$$

For $0 \leqq \lambda \leqq 1$ and $z=r e^{i \theta}$ we have

$$
\begin{aligned}
I(r)= & \int_{0}^{2 \pi}\left|f^{\prime}(z)\right|^{\lambda} d \theta \leqq\left(\frac{r}{\alpha}\right)^{\lambda} \int_{0}^{2 \pi}|F(z)|((1-\alpha) / \alpha) \lambda\left|F^{\prime}(z)\right|^{\lambda} d \theta \\
& +\int_{0}^{2 \pi}|F(z)|^{\lambda / \alpha} d \theta
\end{aligned}
$$

which, on applying Hölder's inequality with conjugate indices $p$ and $q$, yields

$$
\begin{aligned}
I(r) \leqq & \left(\frac{r}{\alpha}\right)^{\lambda}\left[\int_{0}^{2 \pi}|F(z)|((1-\alpha) / \alpha) \lambda p d \theta\right]^{1 / p}\left[\int_{0}^{2 \pi}\left|F^{\prime}(z)\right|^{\lambda q} d \theta\right]^{1 / q} \\
& +\int_{0}^{2 \pi}|F(z)|^{\lambda / \alpha} d \theta .
\end{aligned}
$$

Since $1-\alpha>0$, using (6) and (8) we see that $\lim _{r \rightarrow 1}-I(r)$ exists if $(1-\alpha) \lambda p / \alpha \leqq(1+\epsilon) / 2 \alpha, \lambda q \leqq(1+\epsilon) /(2 \alpha+1+\epsilon)$ and $\lambda / \alpha \leqq(1+\epsilon) / 2 \alpha$, where $1 / p+1 / q=1$ and $p>1$. This system of inequalities will be satisfied with $p=(3+\epsilon) /(2-2 \alpha), q=(3+\epsilon) /(2 \alpha+1+\epsilon)$ and $\lambda$ $\leqq(1+\epsilon) /(3+\epsilon)$.

If we let $\delta=(1+\epsilon) /(3+\epsilon)-1 / 3$ then we have shown that there exists $\delta=\delta(f)>0$ such that $\lim _{r \rightarrow 1^{-}} I(r)$ exists if $\lambda \leqq \delta+1 / 3$, and this completes the proof of (i).

(ii) Since $1-\alpha \leqq 0$ and $\lambda>0$, by using the well-known distortion theorem $\left|f\left(r e^{i \theta}\right)\right| \geqq r\left|f^{\prime}(0)\right| /(1+r)^{2}$, we obtain

$$
|F(z)|^{((1-\alpha) / \alpha) \lambda}=\left|\frac{f(z)}{z}\right|^{(1-\alpha) \lambda} \leqq\left[\frac{\left|f^{\prime}(0)\right|}{(1+r)^{2}}\right]^{(1-\alpha) \lambda} .
$$

Hence (14) becomes 


$$
I(r) \leqq\left(\frac{r}{\alpha}\right)^{\lambda}\left[\frac{\left|f^{\prime}(0)\right|}{(1+r)^{2}}\right]^{(1-\alpha) \lambda} \int_{0}^{2 \pi}\left|F^{\prime}(z)\right|^{\lambda} d \theta+\int_{0}^{2 \pi}|F(z)|^{\lambda / \alpha} d \theta,
$$

and we see from (6) and (8) that $\lim _{r \rightarrow 1^{-}} I(r)$ exists if

$$
\lambda \leqq(1+\epsilon) /(2 \alpha+1+\epsilon) \text { and } \lambda / \alpha \leqq(1+\epsilon) / 2 \alpha .
$$

Let $\delta=(1+\epsilon) /(2 \alpha+1+\epsilon)-1 /(2 \alpha+1)$. Then $\delta=\delta(f)>0$ and $\lim _{r \rightarrow 1^{-}} I(r)$ exists if $\lambda \leqq 1 /(2 \alpha+1)+\delta$. Thus we have shown that $f \in H^{1 /(2 \alpha+1)+\delta}$, and this completes the proof of the theorem.

In the exceptional case when $f \in B\left(\alpha, 0, P, k_{a, \tau}\right)$, we have from (2),

$$
f^{\prime}(z)=a^{\alpha} f(z)^{(1-\alpha)}\left(\frac{1}{1-e^{-i \tau} z}\right)^{2 \alpha} P(z) / z^{1-\alpha} .
$$

Again using Hölder's inequality and the result that $(1-z)^{-1} \in H^{\lambda}$ if and only if $\lambda<1$, we find that (i) if $0<\alpha<1$ then $f^{\prime} \in H^{\lambda}$ for all $\lambda<1 / 3$, and (ii) if $1 \leqq \alpha$ then $f^{\prime} \in H^{\lambda}$ for all $\lambda<1 /(2 \alpha+1)$.

The author is very grateful to Professor F. R. Keogh for his encouragement and advice during the preparation of this paper.

\section{REFERENCES}

1. I. E. Bazilevič, On a case of integrability in quadratures of the Loewner-Kufarev equation, Mat. Sb. 37 (79) (1955), 471-476. (Russian) MR 17, 356.

2. P. J. Eenigenburg and F. R. Keogh, On the Hardy class of some univalent functions and their derivatives, Michigan Math. J. 17 (1970), 335-346.

3. G. H. Hardy and J. E. Littlewood, Some properties of fractional integrals. II, Math. Z. 34 (1932), 403-439.

4. W. K. Hayman, Multivalent functions, Cambridge Tracts in Math. and Math. Phys., no. 48, Cambridge Univ. Press, Cambridge, 1958. MR 21 \#7302.

5. J. E. Littlewood, Lectures on the theory of functions, Oxford Univ. Press, Oxford, 1944. MR 6, 261.

6. A. J. Lohwater, G. Piranian and W. Rudin, The derivative of a schlicht function, Math. Scand. 3 (1955), 103-106. MR 17, 249.

7. C. Pommerenke, Über die Subordination analytischer Funktionen, J. Reine Angew. Math. 218 (1965), 159-173. MR 31 \#4900.

8. D. K. Thomas, On Bazilevič functions, Trans. Amer. Math. Soc. 132 (1968), 353-361. MR $36 \# 5330$.

9. J. Zamorski, On Bazilevič schlicht functions, Ann. Polon. Math. 12 (1962), 83-90. MR 26 \#1446.

10. A. Zygmund, Trigonometrical series. Vol. 1, 2nd ed., Cambridge Univ. Press, New York, 1959. MR 21 \#6498.

University of Kentucky, Lexington, Kentucky 40506 